\title{
3D Mathematics - Seeing is Believing
}

\author{
Ting-Sheng Weng
}

\begin{abstract}
In the 21st Century, an era of knowledge economy, it is necessary to have individuals with abilities in creative thinking and problem-solving. Mathematics cultivates student abilities in independent analysis and deduction. Thus, mathematics is an important required course for high school students, and is a valuable tool for application to phenomena in the natural world. It is a science for learning to handle and solve problems of change, which enhances student computational abilities, abstract thinking, logical deduction abilities, and spatial imagination, while helping students to gain training toward resolving actual problems. This study applied a program design to a calculus course, and used $3 D$ dynamic models to allow students to describe and observe objects from various angles in order to enhance their interest and understanding of mathematics. From the integration of applications of mathematical formulas, computer programming, design, and imaging $3 \mathrm{D}$ mathematics, with the processes of constructing model objects in 3D visualization for mathematical formulas, it would be possible to train talent capable of industrial response, management, and value creation.
\end{abstract}

Index Terms-Python, 3D mathematics, program design, 3D, mathematics, calculus, seeing is believing, digital learning, creation of value.

\section{INTRODUCTION}

In the 21st Century, an era of knowledge economy, it is necessary to have individuals with abilities in creative thinking and problem-solving. Mathematics provide training for comprehensive independent analysis and deduction ability for students, and if extended to applied mathematics, statistical sciences, physics, information science, management curricula and courses, and accommodate basic mathematics courses, it can be used to provide training for students in diverse future career planning.

Mathematics is an important required course for high school students, which allows students to gain basic knowledge and theoretical foundations for vector algebra, spatial analytic geometry, and calculus. Moreover, mathematics can cultivate students' calculations, abstract thinking, logical deduction, and spatial imaginative abilities. Training in practical problem-solving abilities lays a foundation for students of subsequent courses. Mathematical knowledge applications can be extended into other fields, especially in the sciences.

Manuscript received March 15, 2011.

Ting-Sheng Weng. Hi is an Assistant Professor of the department of Business Administration at National Chiayi University, \#580 Sinmin Road, Chiayi City 600, Taiwan. He received his Ph. D. in Division of management and information science, Graduate School of Advanced Technology from Kinki University, Japan in 2000. He had served as visiting Professor at Meiji University in Japan from 2006 through 2007. His major research interests include science technology and management, e-learning, MIS, and image processing. (e-mail: politeweng@mail.ncyu.edu.tw).

\section{RESEARCH Motives AND PuRPoses}

In undergraduate studies, calculus is an important course for advanced mathematics. However, the current instruction and study of calculus are limited to the deduction of formula theories and problem-solving, while neglecting students' innovative thinking and practical application abilities. The students especially lack the conceptual abilities required for calculus principles and the physical representation of formulas. The prevalence of multimedia has led to poor abstract thinking abilities and frequent use of 3D figures and images in learning. In order to help students employing theories to solve practical problems, and thus, enhance their learning interest in mathematics, this study aimed to apply an application-oriented program to design a digital, multimedia teaching program for a calculus course, which uses 3D images to explain dimensional concepts. This is used to strengthen student abilities in mathematical calculations, digitized data management, program design, dynamic 3D observations, and understanding, in order to conform to and cope with existing demands in contemporary diverse societies, and provide a more competitive edge in industrial markets.

\section{LITERATURE REVIEW}

Globalization and liberalization have led to diverse product demands, while rapid developments of new technologies and new techniques have led to the advent of an information economy age (Hargreaves, 2000; Lee, 2000). An individual's knowledge is a primary asset, which is an important force behind personal futures, corporate wealth, and national economies. The combination of knowledge and technology has become a primary source of national competitiveness, while the application of knowledge and information can be combined with existing industrial and core abilities to increase international competitiveness and competency. Education should provide core knowledge resources to cultivate innovative abilities and promote learning in order to strengthen student creative design and practical ability (Ministry of Education, 2000; Wu Ching-shan, 2004). The learner continues to absorb and gain through knowledge and practical application, allowing them to create new knowledge and practical applications.

\section{A. Response abilities create value}

Individuals or members of a technical team with rich and diverse knowledge and experience would have broader cognition abilities, allowing them to understand and explain more diverse types of information, and thus, be more capable of resolving difficult problems (Daft and Weick, 1984) with better response abilities. The absorbing ability of an organization is based on individual learning abilities within an organization (Cohen \& Levinthal, 1990), and 
personal learning ability is based on past knowledge and experience. As students gain knowledge and accumulate experience, it is important to provide them with the breadth and depth of knowledge and techniques, as this facilitates interpretation and an ability to absorb and convert knowledge information into tangible or intangible products, which are beneficial toward innovative performance, thus, creating value.

\section{B. Comprehension ability for mathematics}

Liu (1998) conducted a study on freshman students at the National Taipei University of Education, National Chia-yi University and the National Pingtung University of Education in order to investigate their comprehension of mathematics [1]. Results showed that, in terms of numbers and mathematics, they failed to understand the basic concepts and meanings of whole number multiplication and division; in measurements and testing, they overlooked the rationality of units and the appropriateness of formula use; in terms of figures and space, they were unclear on the concepts on parallelogram surfaces, line symmetry, prism bases, and expanded cone diagrams; and in terms of statistical graphs, they were unclear on the differences between, and applications of, bar graphs, line graphs, and pie graphs. Lin (2003) surveyed students at the National Hsinchu University of Education, and found that, they have insufficient understanding regarding indicators of mathematical abilities [2], as only about $18 \%$ could understand more than $70 \%$ of the indicators, suggesting a serious lack of mathematical knowledge. The shortcoming of textbooks is that they cannot use external dynamic expressive methods to interpret abstract concepts; and students learning only from mathematics textbooks may experience some degree of learning difficulty from reading only textbooks. In 1987, a National Science Teacher Association (NSTA) study found that, using computers and software to teach can elevate student learning motivations, student cooperation, independence, and opportunities for elevating learning abilities. Evidently, the usage of suitable computers and related software can influence student learning contexts to obtain satisfactory results in cognition and emotional learning accomplishments (Hsieh Che-jen, Chen Hong-pin, 2008). The National Council of Teachers of Mathematics (NCTM, 1989) pointed out that, while teaching, teachers should use concrete visual models, and technological products in a timely manner. Computer instruction can elicit another kinds of interest in students for learning mathematics. If teachers can create visual models, where abstract mathematical symbols can be converted into real objects that students can see and concretely manipulate, it is believed that such activities could produce more significant effects on the construction of mathematical concepts for students.

Markovits, Eylon \& Bruckheimer (1988) suggested that images are visual representations, and a display of images of functions can help with observations and discussions of the characteristics of functions, which facilitate teacher instruction and help learners to learn. Computer computations can use dynamic images to provide learners with powerful learning and perceptive experiences, and in turn help learners form internal representations with dynamic connections, providing abstract concepts with a basis in perception (Hsieh Che-jen, 2002).

According to Hsieh, Shiu, Liu, Lu and Chen (2004), 50\% of teachers think that "software tools to assist learning can increase the students' willingness to learn" and "lack of software tools to assist learning” may be a reason for poor learning attitudes in students [3]. Moreover, 39\% of teachers agree that "instructional methods cannot elicit student's interest in learning”, and 53\% suggest that "the learning methods preferred by students have changed, while instructional methods have not adjusted or changed accordingly" is the reason that students have poor learning attitudes. This shows that more than half of teachers agree with the necessity of changing instructional methods. In addition, $72 \%$ of teachers think that it would be helpful to "use instructional animation that shows the process of solving example problems suitable to the instructional materials.” In mathematics courses, $47 \%$ of teachers think that current mathematics-related courses "lack suitable computer-assisted tools” for instruction, while 56\% think that "the content of instructional materials is not interesting or lively", 56\% agree that "the lack of software tools to assist learning" may cause poor learning attitudes in students, and $58 \%$ think that it is necessary to "use computer-assisted tools in selected mathematics courses." At the same time, most teachers believe that computer-assisted instructional tools could visually portray and concretely explain theoretical mathematical knowledge. With regard to students, $56 \%$ agree that "textbook content is insufficiently interesting". Moreover, 37\% think that "lack of software tools to assist learning" cause difficulties in learning, which reflects a certain degree of importance and necessity in developing mathematical learning software; $50 \%$ of the students believe that they need computer-assisted tools to learn mathematics; while nearly 80\% agree that computer-assisted tools can help to acquire knowledge in mathematics courses. Among them, students propose that suitable usage of mathematical assistance tools are conducive in verifying assignments and clarifying concepts, and simplifying repetitive calculation processes, allowing the focus to be centered on understanding mathematical concepts. Therefore, it is necessary to develop visualized 3D mathematics to assist in learning.

\section{DEVELOPMENT TOOLS}

Instruction through a computer is livelier than traditional instruction, and can better elicit student interest. If teachers can create visual models for abstract mathematical symbols to be converted into objects that students can see and concretely operate, it would certainly cause the construction of mathematical concepts to become much easier and more effective.

GeoGebra, Maxima, Matlab, Mathematica, MathCAD, and Flash can be used to create dynamic models for calculus.

GeoGebra is a dynamic geometry freeware system, in which points, vectors, line segments, straight lines, conic section, and functions can be used as diagrams, upon which dynamic adjustments can be made. There can be direct input 
for equations and coordinates, numbers, vectors, and variables of points. It can also solve the differentials or integrals of functions, find the roots of equations, maximum and minimum functions, and provide commands such as Root and Extremum; moreover, the equations in the algebra windows correspond to the geometric diagrams, which mean variables are considered. Maxima is a freeware algebra system that can be applied to differential and integral calculus, matrix calculations, 2D, and 3D diagrams, as well as calculate trigonometric functions, calculus, linear algebra, and others. MATLAB is a computer language, in which there are discussion functions, programming, and execution abilities; it is a platform capable of scientific algorithms, and can draw 3D images, animation, and engage in image rendering of different types of figures. Mathematica combines a calculation engine for values and signs, an image system, a programming language, and a text system, which can describe mathematical and scientific concepts with high-level links to other programs. MathCAD is an interactive value calculation system; when a formula, equation group, or matrix is input, the calculator can directly give the results without concern of the calculation process. Thus, MathCad is used for complex mathematical calculations, image displays, and text file handling of many technological fields. Flash is a software designed for program development, and has become the most popular software platform, as it is used on $98 \%$ of computers when connecting to the Internet. In various operation systems and browsers, Flash can provide the same media experience, which is the advantage of using Flash on the Internet. Flash technology only requires one-time coding and production, can integrate various types of media applications, and be used to produce fine animation. Action Script is used to write programs for interactions and logic processing in order to quickly control various multimedia resources.

When developing a 3D calculus tool, this study used the cross-platform code Python for program design, which is an object-oriented, direct-translation computer language that provides good support for $\mathrm{C}$ and other languages. It is a language with a wide range of applications.

\section{3D VISUALIZATION APPLIED IN INSTRUCTION}

The application of 3D technology in instruction has become a popular trend, as 3D instructional materials can effectively convey curricular content (Huang and Liao, 2008) [4]. A former software engineer, Adam Finkelstein (1998), used a computer to simulate actual items, and pointed out that the conception and creation of calculating visual designs requires mathematics and computerized drawing [5], as such programs are used to logically and structurally convey virtual visual concepts onto a screen. Visualizing equations is beneficial for multifaceted inspiration in media design and visual design.

This study used Python and 3D Vpython to design 3D images. The image for the differential calculus equation $\mathrm{z}=\sin \left(\mathrm{x}^{\wedge} \_\mathrm{y}^{\wedge} 2\right)$ is shown in Figure1:

\section{INSPIRE CONTROL AND RESPONSE ABILITY}

$3 \mathrm{D}$ visualization technology provides users with visual perceptions and feelings that approach reality, and allows them to freely manipulate visual points and view directions within a 3D environment, resulting in greater understanding, such as from Figure1a to 1 .Visualized 3D figures can also be utilized in diverse extended designs and concrete

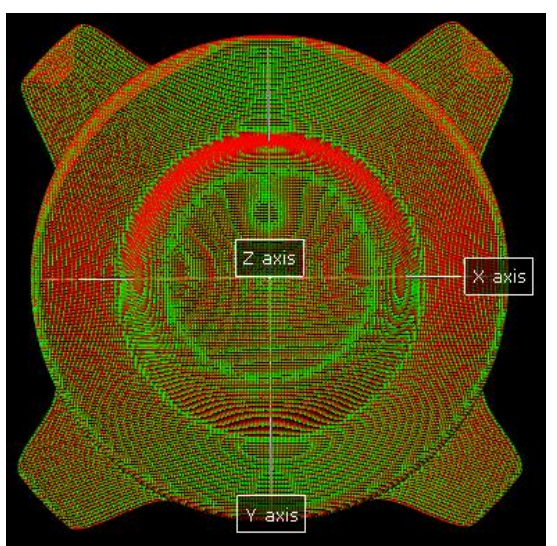

(a)

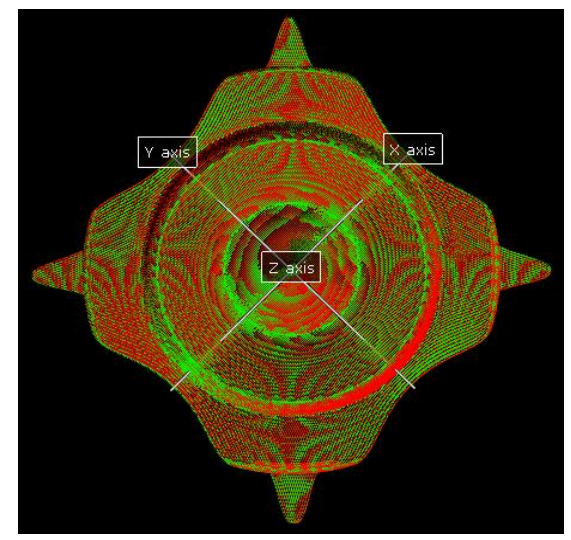

(d)

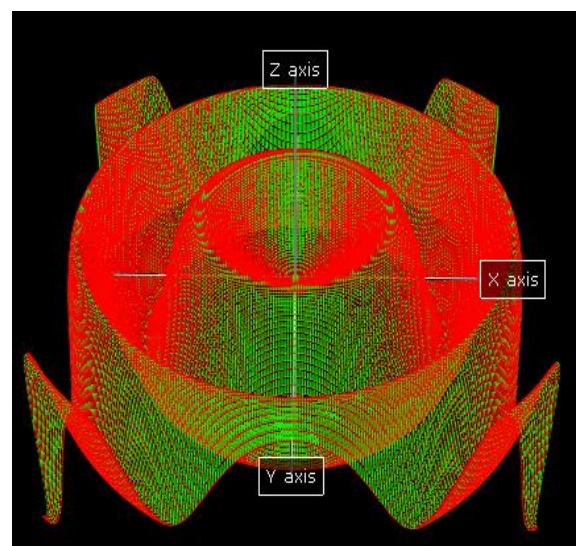

(b)

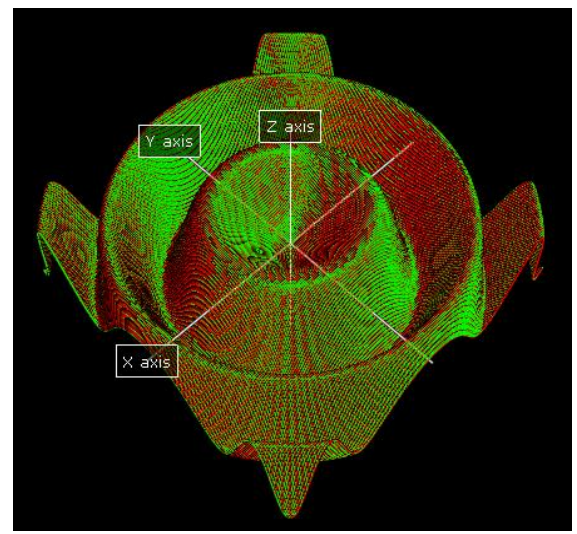

(e)

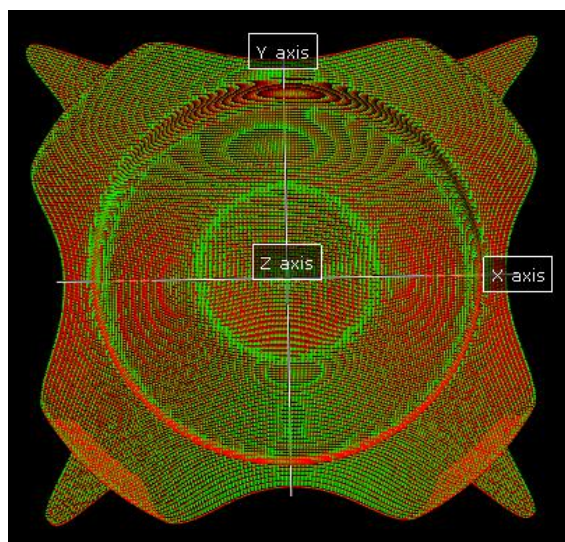

(c)

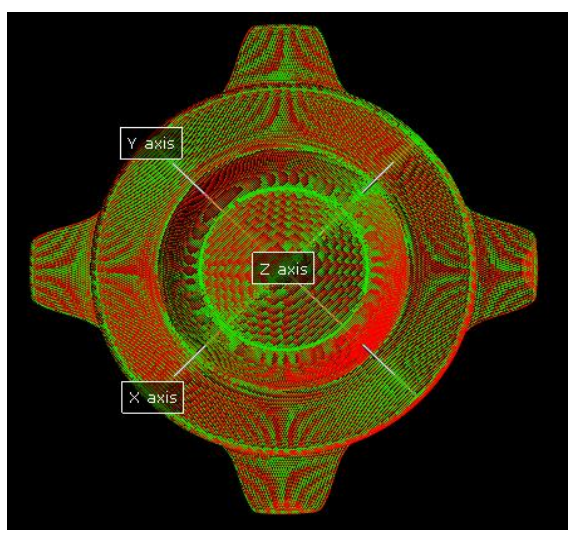

(f) 


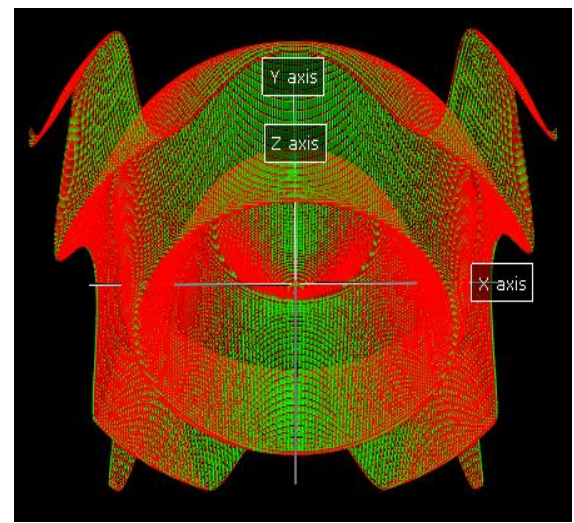

(g)

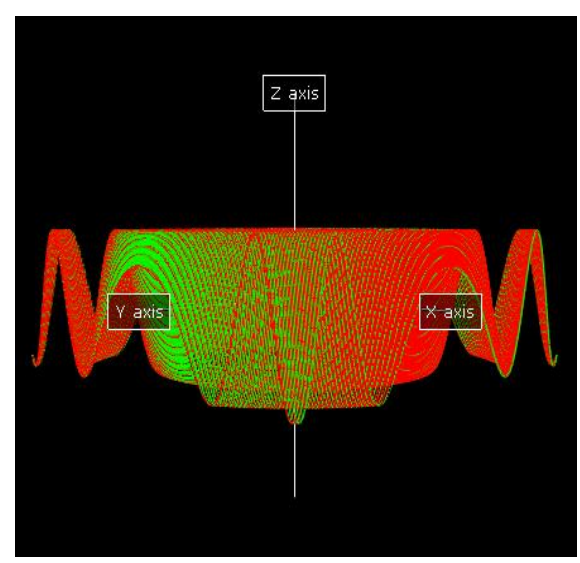

(j)

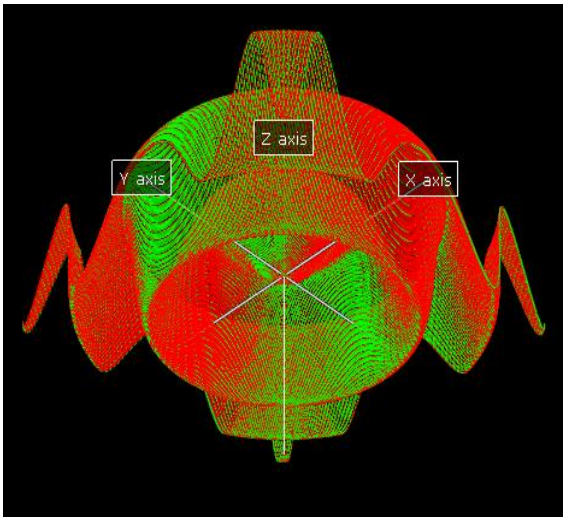

(h)

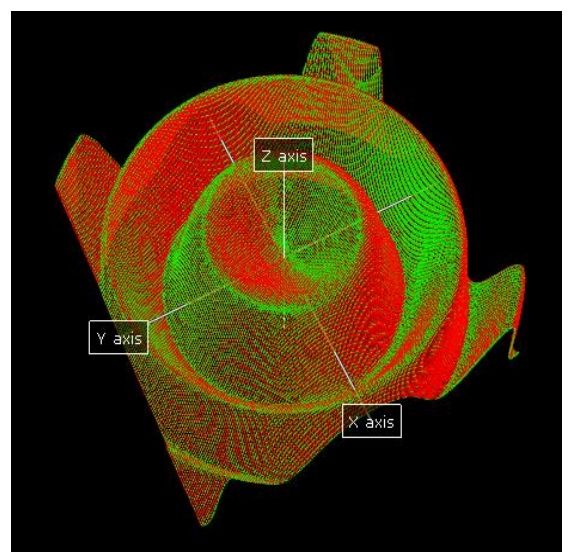

(k)

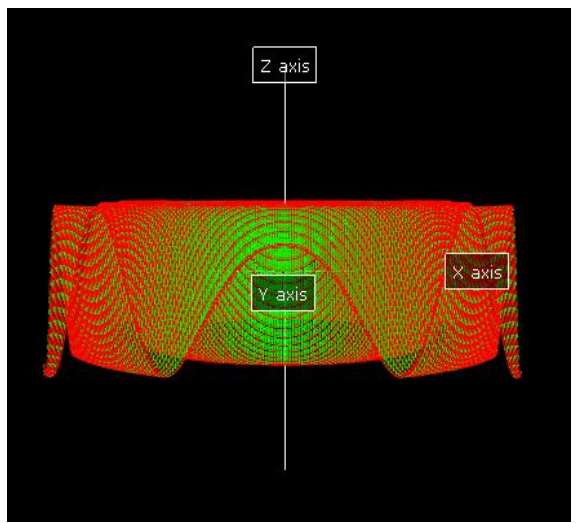

(i)

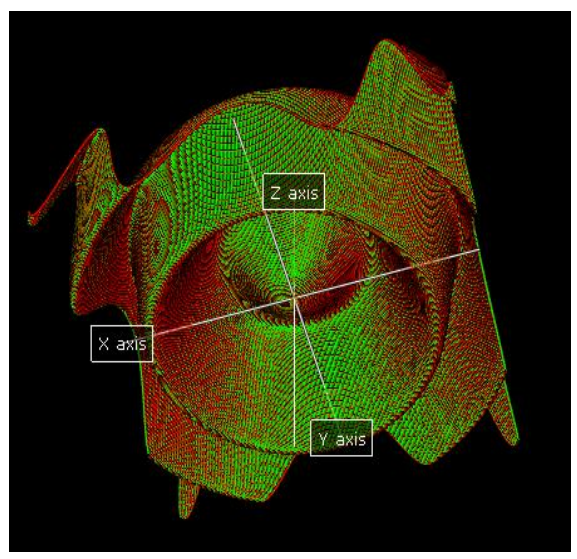

(l)

Figure1. Observing changes of dynamic images from different angles, which can be freely spun from Diagram a to Diagram 1

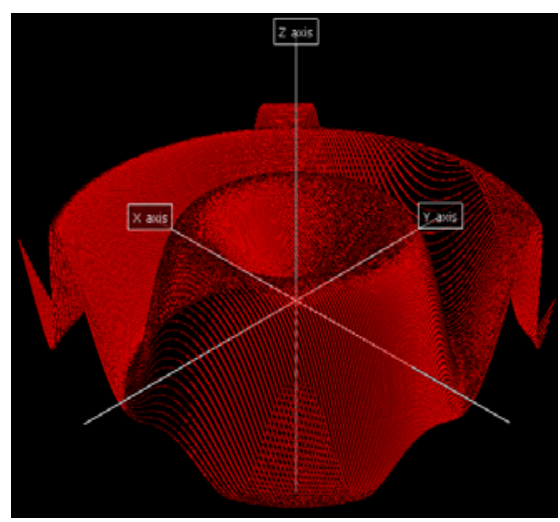

(m)

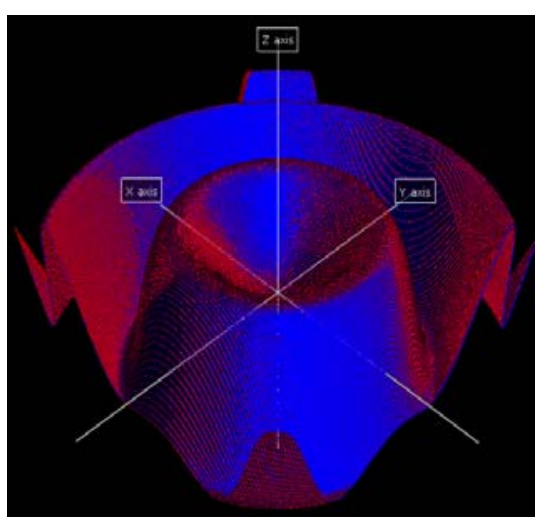

(n)

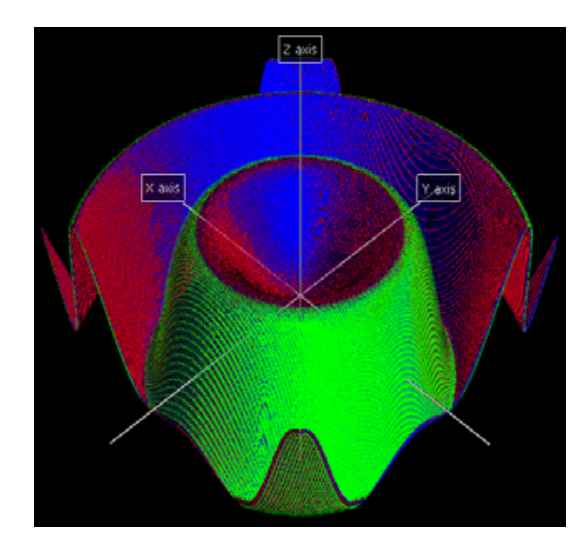

(p)

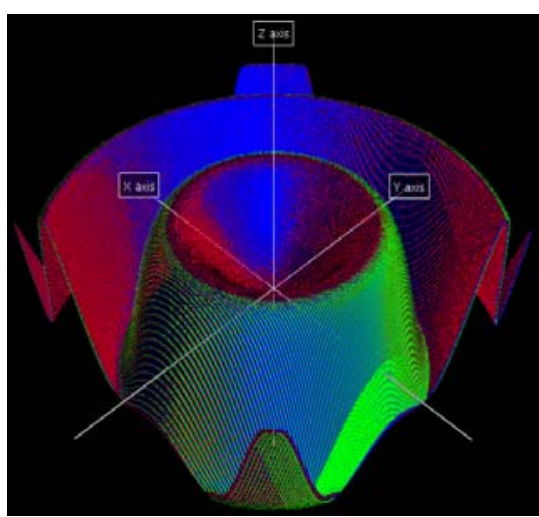

(o)
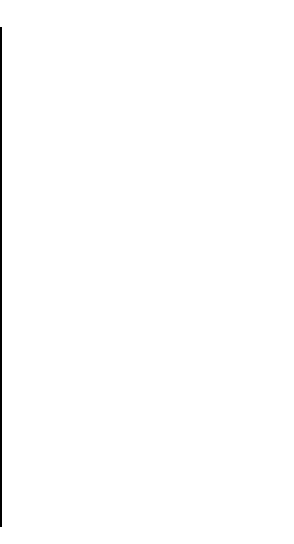
have their own preferred lucky colors. In the Americas, red, green, blue, and black are generally matched together, European countries tend to lean more towards blue, while Asian countries, China, India, Thailand, Mongolia, Taiwan, Singapore, and Hong Kong prefer red. Islamic, Indian, and Egyptian countries tend to like green. For instance in Figure 2 , it is possible to train students to cope with customized demands for customers of international markets, with design and technical management abilities in colors and images.

\section{CONCLUSIONS}

The visible and visual 3D dynamic design, as proposed by this study, could help to enhance learning interests and effects of mathematics courses, improve students' interdisciplinary and multimedia design abilities, as well as foster a technological learning ability, facilitating them to become professional talents in integrating mathematical principles and information. In the process of learning, they can also obtain abilities in thinking, understanding, controlling, responding, and ad creation.

Future studies can continue to research and develop a calculus dynamic digital learning system that uses multimedia and interactive tools, and apply such a design to course designs, instructional materials, constructing learning environments, online supplementary materials, online tests, and evaluation systems, as well as a virtual multimedia mathematics teaching platform. The results will be provided to teachers for computer-assisted instruction, as well as to learners to enhance learning initiatives.

\section{ACKNOWLEDGEMENT}

The author appreciates the comments of the review committee. This project was supported by NSC (National Science Council) Taiwan under grant number: NSC 99-2511-S-415 -004.

The authors would like to thank Prof. De-ching Yang,
Prof. Meng-Hui Hsu and Prof. Ren-yan Lin for their help and support.

\section{REFERENCES}

[1] D. Hargreaves, Knowledge management in the learning society. Retrieved January, 12, 2001, from http://www.oecd.org/pdf/ M00026000/ M00026245.pdf

[2] J. Lee, Sr., "Knowledge management: The intellectual revolution,” IIE Solutions, Oct. 2000, pp.34-37.

[3] Ministry of Education, Higher Education Bulletin - National Knowledge Economy Development Convention, Nov. 2000.

[4] C.S. Wu, On School Promotion of Knowledge Management Strategies - Elementary Schools as An Example, NSC92-2413-H-133-003, 2004, pp.1-208.

[5] R.I. Daft, and K. E. Weick, Toward a Model of Organizations as Interpretation Systems, Academy of Management Review, 9:2, 1984, pp. 284-295.

[6] M.L. Liu, Elementary School Mathematical Knowledge of Education College Students. Papers from Academic Conference on Education, 1998, pp.883-917.

[7] P.Y. Lin, "Discussing Elementary School Coping Strategies from the Perspective of Training Grade 1-9 Curriculum Mathematics Teachers,” Primary Education, Vol. 208, 2003, pp.73-84.

[8] C.J. Hsieh, and H.P. Chen, Ministry of Education Plan for Subsidies for Professional Schools to Elevate Overall Instructional Quality Plan for Comprehensive Enhancement of Instructional Quality in General Education for National Tainan Institute of Nursing, 2008, pp.1-47.

[9] C.J. Hsieh, Design Examples and Theoretical Explorations in Dynamic Computer Geometry Instruction Construction, Graduate Institute of Mathematic Education, National Chia Yi University: Reforming Mathematics Education Issues in Elementary and Junior High Schools, 2002, pp. 225-244, Kaohsiung: Fuwen Publishing.

[10] S.H. Hsieh, S.Y. Shiu, K.F. Liu, L.C. Lu, C.S. Chen, Planning, Design, and Development of Web-Based Computer-Aided Integrated Courses on Engineering Mechanics and Mathematics, National Science Council, NSC92-2516-S002-008, 2004.

[11] Z. Markovits, B. Eylon, and M. Bruckheimer, Difficulties students have with the function concept. In A.F. Oxford \& A. P. Shulte (Ed.), The idea of algebra, 1988, pp. 43-60, Reston, VA: National Council of Teachers of Mathematics.

[12] H.M. Huang, S.C. Liao, Constructing and Evaluating 3D Virtual Reality in Teaching, National Science Council, NSC96-2628-S025-001-MY2, 2008.

[13] A. Finkelstein, Adam Finkelstein,s cool images, 1998, Available at http ://wwww.cs.princeton.edu/ af/cool /inde dex.html. 